LINEAR AND NON-LINEAR THEORY OF

GENERALIZED FUNCTIONS AND ITS APPLICATIONS

BANACH CENTER PUBLICATIONS, VOLUME 88

INSTITUTE OF MATHEMATICS

POLISH ACADEMY OF SCIENCES

WARSZAWA 2010

\title{
LAPLACE ULTRADISTRIBUTIONS SUPPORTED BY A CONE
}

\author{
SŁAWOMIR MICHALIK \\ Faculty of Mathematics and Natural Sciences, College of Science \\ Cardinal Stefan Wyszyński University \\ Dewajtis 5, 01-815 Warszawa, Poland \\ E-mail: s.michalik@uksw.edu.pl
}

\begin{abstract}
The space of Laplace ultradistributions supported by a convex proper cone is introduced. The Seeley type extension theorem for ultradifferentiable functions is proved. The Paley-Wiener-Schwartz type theorem for Laplace ultradistributions is shown. As an application, the structure theorem and the kernel theorem for this space of ultradistributions are given.
\end{abstract}

1. Introduction. The theory of ultradistributions introduced by C. Roumieu [7] and A. Beurling [1] is a natural generalisation of the theory of Schwartz distributions. It was systematically investigated by H. Komatsu in his fundamental papers [3] and [4]. In particular he proved the structure theorem and the kernel theorem for the space $D^{\prime\left(M_{p}\right)}(\Omega)$ of ultradistributions. Moreover he described the image of this space under Fourier-Laplace transformation (the Paley-Wiener-Schwartz type theorem).

In a similar way G. Eysik [5] introduced and studied the space $L_{(\omega)}^{\prime\left(M_{p}\right)}\left(\Gamma_{v}\right)$ of Laplace ultradistributions of Beurling type supported by a half line $\Gamma_{v}:=[v, \infty)$. We emphasise that this space of ultradistributions is convenient for the study of Laplace transformation.

On the other hand, in the theory of the hyperbolic equations it is convenient to consider distributions and ultradistributions supported by a cone (see Chapter III in [9]).

Hence it seems to be natural to introduce the space of Laplace ultradistributions supported by a cone. In the paper we define and study this space for closed convex fat proper cones in $\mathbb{R}^{n}$ following G. Łysik's approach.

The paper is organised as follows. In the first sections we introduce the notations and the notions of cones and of ultradistributions.

2000 Mathematics Subject Classification: Primary 46F05, 46F12, 44A10.

Key words and phrases: ultradistributions, extension theorem, Laplace transform, kernel theorem. The paper is in final form and no version of it will be published elsewhere. 
Section 4 contains one of the main results of the paper - the Seeley type extension theorem for Laplace ultradifferentiable functions supported by a cone. As an application we show completeness of this space of functions.

The second main result - the Paley-Wiener-Schwartz type theorem for Laplace ultradistributions supported by a cone - is given in Section 5. Following G. Eysik [5], as a corollary we obtain the structure theorem for this space of ultradistributions.

As an application, in the last section we show the version of the kernel theorem. In this way we generalise our earlier result [6] to the convex proper cones.

2. Notation and cones in $\mathbb{R}^{n}$. Let us denote by $\|\cdot\|$ the Euclidean norm in $\mathbb{R}^{n}$ (or $\left.\mathbb{C}^{n}\right)$. We set $\langle x\rangle=1+|x|$ for $x \in \mathbb{R}$ and similarly $\langle z\rangle=1+|z|$ for $z \in \mathbb{C}$.

For any $\varepsilon>0$ and $S \subseteq \mathbb{R}^{n}$ let $(S)_{\varepsilon}$ denote the set $\left\{x \in \mathbb{R}^{n}: \operatorname{dist}(x, S) \leq \varepsilon\right\}$. $\mathbb{S}^{n}$ denotes the unit $n$-sphere in $\mathbb{R}^{n+1}$.

Let us consider $\Gamma \subseteq U \subseteq \mathbb{R}^{n}$, such that $U$ open in $\mathbb{R}^{n}, \Gamma$ is relatively closed in $U$ and $\Gamma \subseteq \overline{\operatorname{Int} \Gamma}$ (i.e. $\Gamma$ is a fat set). Then for $k \in \mathbb{N}_{0} \cup\{\infty\}$ we set

$$
C^{k}(\Gamma):=\left\{f: \Gamma \rightarrow \mathbb{C} \mid \text { there exists } g \in C^{k}(U) \text { such that }\left.g\right|_{\Gamma}=f\right\} .
$$

We write $D$ for the differential operator $\frac{d}{d x}$.

Let $\left\{P_{\tau}\right\}_{\tau \in T}$, be a family of vector spaces. Then $\underset{\tau \in T}{\lim } P_{\tau}\left(\right.$ resp. $\left.\underset{\tau \in T}{\lim } P_{\tau}\right)$ denotes the inductive limit (resp. projective limit) of $P_{\tau}, \tau \in T$.

$A$ cone in $\mathbb{R}^{n}$ (with vertex at 0 ) is a set $\Gamma \subseteq \mathbb{R}^{n}$ with the property that if $x \in \Gamma$, then also $\lambda x \in \Gamma$ for all $\lambda>0$.

If $\Gamma$ is a cone in $\mathbb{R}^{n}$ (with vertex at 0 ) and $v \in \mathbb{R}^{n}$ then the set $\Gamma_{v}:=\Gamma+v$ is called a cone in $\mathbb{R}^{n}$ with vertex at $v$.

For a given cone $\Gamma$ in $\mathbb{R}^{n}$ (with vertex at 0 ), the set

$$
\Gamma^{*}:=\{\xi: \quad \xi \cdot x \geq 0, \forall x \in \Gamma\}
$$

is said to be dual to $\Gamma$. The same set $\Gamma^{*}$ is also called dual to $\Gamma_{v}=\Gamma+v$.

Observe that $\Gamma^{*}$ is a closed convex cone with vertex at 0 (see [9]). If, in addition, Int $\Gamma^{*} \neq \emptyset$ then a cone $\Gamma$ (or, generally, a cone $\Gamma_{v}$ ) is called proper.

We say that $x$ is less than $y$ in $\Gamma$-sense if $y-x \in \operatorname{Int} \Gamma$; we then write $x<_{\Gamma} y$. Similarly we write $x \leq_{\Gamma} y$ if $y-x \in \bar{\Gamma}$.

3. Laplace ultradistributions. Let $\left(M_{p}\right)_{p \in \mathbb{N}_{0}}$ be a sequence of positive numbers satisfying the conditions (see [3]):

(M.0) $M_{0}=M_{1}=1$;

(M.1) (logarithmic convexity) $M_{p}^{2} \leq M_{p-1} M_{p+1}$ for $p \in \mathbb{N}$;

(M.2) (stability under ultradifferential operators) there are constants $A, H$ such that

$$
M_{p} \leq A H^{p} \min _{0 \leq q \leq p} M_{q} M_{p-q} \text { for } p \in \mathbb{N}_{0}
$$

(M.3) (strong non-quasi-analyticity) there is a constant $A$ such that

$$
\sum_{q=p+1}^{\infty} \frac{M_{q-1}}{M_{q}} \leq A p \frac{M_{p}}{M_{p+1}} \quad \text { for } \quad p \in \mathbb{N}_{0} .
$$


Let $m_{p}:=M_{p} / M_{p-1}$ for $p \in \mathbb{N}$. Observe that (M.1) is equivalent to saying that the sequence $m_{p}$ is non-decreasing. By (M.3) we have $m_{p} \rightarrow \infty$.

The associated function of the sequence $\left(M_{p}\right)$ is defined by $M(\rho):=\sup _{p \in \mathbb{N}_{0}} \ln \frac{\rho^{p}}{M_{p}}$ for $\rho>0$.

An ultradifferential operator $P(D)$ of class $\left(M_{p}\right)$ is defined by $P(D):=\sum_{\alpha \in \mathbb{N}_{0}^{n}} a_{\alpha} D^{\alpha}$, where $a_{\alpha} \in \mathbb{C}$ satisfy the following condition: there are constants $K<\infty$ and $C<\infty$ such that $\left|a_{\alpha}\right| \leq C \frac{K^{|\alpha|}}{M_{|\alpha|}}$ for $\alpha \in \mathbb{N}_{0}^{n}$.

The entire function $\mathbb{C}^{n} \ni z \mapsto P(z):=\sum_{\alpha \in \mathbb{N}_{0}^{n}} a_{\alpha} z^{\alpha}$ is called a symbol of class $\left(M_{p}\right)$.

We will introduce some spaces of ultradifferentiable functions and of ultradistributions.

Definition 3.1 (see [3]). Let $\Omega$ be a fat set in $\mathbb{R}^{n}$. The space $\mathcal{E}^{\left(M_{p}\right)}(\Omega)$ of ultradifferentiable functions on $\Omega$ of class $\left(M_{p}\right)$ is defined as

$$
\mathcal{E}^{\left(M_{p}\right)}(\Omega):=\lim _{K \subset \subset \subset} \mathcal{E}^{\left(M_{p}\right)}(K)
$$

where for any compact subset $K$ of $\Omega$

$$
\mathcal{E}^{\left(M_{p}\right)}(K):=\lim _{h>0} \mathcal{E}_{h}^{\left(M_{p}\right)}(K)
$$

and for any $h>0$

$$
\mathcal{E}_{h}^{\left(M_{p}\right)}(K):=\left\{\varphi \in C^{\infty}(K):\|\varphi\|_{K, h}^{\left(M_{p}\right)}:=\sup _{y \in K} \sup _{\alpha \in \mathbb{N}_{0}^{n}} \frac{\left|D^{\alpha} \varphi(y)\right|}{h^{|\alpha|} M_{|\alpha|}}<\infty\right\} .
$$

Definition 3.2 (see [3]). Let $\Gamma_{v}$ be a closed fat cone in $\mathbb{R}^{n}$. The space $D^{\prime\left(M_{p}\right)}\left(\Gamma_{v}\right)$ of ultradistributions on $\Gamma_{v}$ of class $\left(M_{p}\right)$ is defined as the dual space of

$$
D^{\left(M_{p}\right)}\left(\Gamma_{v}\right):=\lim _{\substack{\longrightarrow \subset \subset \Gamma_{v} \\ \lim _{h>0}}} D_{K, h}^{\left(M_{p}\right)}\left(\Gamma_{v}\right),
$$

where for any compact subset $K$ of $\Gamma_{v}$ and any $h>0$

$$
D_{K, h}^{\left(M_{p}\right)}\left(\Gamma_{v}\right):=\left\{\varphi \in C^{\infty}\left(\Gamma_{v}\right): \operatorname{supp} \varphi \subset K \text { and }\|\varphi\|_{K, h}^{\left(M_{p}\right)}:=\sup _{y \in K} \sup _{\alpha \in \mathbb{N}_{0}^{n}} \frac{\left|D^{\alpha} \varphi(y)\right|}{h^{|\alpha|} M_{|\alpha|}}<\infty\right\} .
$$

Definition 3.3 (see [5]). Let $\omega \in(\mathbb{R} \cup\{\infty\})^{n}$ and let $\Gamma_{v}$ be a closed fat cone in $\mathbb{R}^{n}$. The space $L_{(\omega)}^{\prime\left(M_{p}\right)}\left(\Gamma_{v}\right)$ of Laplace ultradistributions supported by a cone $\Gamma_{v}$ is defined as the dual space of

$$
L_{(\omega)}^{\left(M_{p}\right)}\left(\Gamma_{v}\right):=\lim _{\substack{a<\Gamma^{*} \omega}} L_{a}^{\left(M_{p}\right)}\left(\Gamma_{v}\right)
$$

where for any $a<_{\Gamma^{*}} \omega$

$$
L_{a}^{\left(M_{p}\right)}\left(\Gamma_{v}\right):=\lim _{h>0} L_{a, h}^{\left(M_{p}\right)}\left(\Gamma_{v}\right)
$$

and for any $h>0$

$$
L_{a, h}^{\left(M_{p}\right)}\left(\Gamma_{v}\right):=\left\{\varphi \in C^{\infty}\left(\Gamma_{v}\right):\|\varphi\|_{\Gamma_{v}, a, h}^{\left(M_{p}\right)}:=\sup _{y \in \Gamma_{v}} \sup _{\alpha \in \mathbb{N}_{0}^{n}} \frac{\left|e^{-a \cdot y} D^{\alpha} \varphi(y)\right|}{h^{|\alpha|} M_{|\alpha|}}<\infty\right\} .
$$

Observe that the space $L_{(\omega)}^{\left(M_{p}\right)}\left(\Gamma_{v}\right)$ is nonempty if and only if $\Gamma_{v}$ is a proper cone. In this case we have 
Lemma 3.4. Assume that $\Gamma_{v}$ is a closed fat proper cone in $\mathbb{R}^{n}$. Then $D^{\left(M_{p}\right)}\left(\Gamma_{v}\right)$ is a dense subspace of $L_{(\omega)}^{\left(M_{p}\right)}\left(\Gamma_{v}\right)$. Thus $L_{(\omega)}^{\prime\left(M_{p}\right)}\left(\Gamma_{v}\right)$ is a subspace of the space $D^{\prime\left(M_{p}\right)}\left(\Gamma_{v}\right)$ of ultradistributions.

Proof. Making appropriate translation we can assume that $\Gamma_{v}=\Gamma$ is a cone with vertex at 0 . Let $\varphi \in L_{(\omega)}^{\left(M_{p}\right)}(\Gamma)$. Then there exist $a<_{\Gamma^{*}} b<_{\Gamma^{*}} \omega$ such that $\varphi \in L_{a}^{\left(M_{p}\right)}(\Gamma) \subset$ $L_{b}^{\left(M_{p}\right)}(\Gamma)$. By the Denjoy-Carleman-Mandelbrojt theorem (see Theorem 4.2 in [3]) there exists a function $\psi \in D^{\left(M_{p}\right)}(\Gamma)$ such that $0 \leq \psi(y) \leq 1$ for $y \in \Gamma, \psi(y)=1$ for $\|y\| \leq 1$ and $\psi(y)=0$ for $\|y\| \geq 2$. Put

$$
\varphi_{\nu}(y):=\psi\left(\frac{1}{\nu} y\right) \varphi(y) \quad \text { for } y \in \Gamma \quad \text { and } \nu \in \mathbb{N} .
$$

Then $\varphi_{\nu}(y) \in D^{\left(M_{p}\right)}\left(\Gamma_{v}\right)$. Analogously to Lemma 3.4 in [5] we obtain that

$$
\varphi_{\nu} \rightarrow \varphi \quad \text { in } L_{b}^{\left(M_{p}\right)}(\Gamma) \quad \text { as } \nu \rightarrow \infty
$$

and the proof is complete.

EXAMPLE 3.5. The function

$$
\Gamma_{v} \ni y \mapsto \exp _{z} y:=e^{y \cdot z}
$$

belongs to $L_{(\omega)}^{\left(M_{p}\right)}\left(\Gamma_{v}\right)$ if and only if $\operatorname{Re} z<_{\Gamma^{*}} \omega$. Moreover, for any $a<_{\Gamma^{*}} \omega$ and $h>0$ we have

$$
\begin{aligned}
\left\|\exp _{z}\right\|_{\Gamma_{v}, a, h}^{\left(M_{p}\right)} & =\sup _{y \in \Gamma_{v}} \sup _{\alpha \in \mathbb{N}_{0}^{n}} \frac{\left|e^{-a \cdot y} z^{\alpha} e^{y \cdot z}\right|}{h^{|\alpha|} M_{|\alpha|}} \leq e^{(\operatorname{Re} z-a) \cdot v} \sup _{\alpha \in \mathbb{N}_{0}^{n}} \frac{\left|z^{\alpha}\right|}{h^{|\alpha|} M_{|\alpha|}} \\
& =e^{(\operatorname{Re} z-a) \cdot v} \exp M\left(\frac{\left|z_{1}\right|}{h}\right) \cdots \exp M\left(\frac{\left|z_{n}\right|}{h}\right)<\infty
\end{aligned}
$$

with $\operatorname{Re} z<_{\Gamma^{*}} a$.

Definition 3.6. For $a \in \mathbb{R}^{n}$ and $\omega \in(\mathbb{R} \cup\{\infty\})^{n}$ we define

$$
Y_{a}:=\operatorname{span}\left\{\exp _{c}\right\}_{c \Gamma_{\Gamma^{*}} a}, \quad Y_{(\omega)}=\bigcup_{a<\Gamma^{*} \omega} Y_{a} .
$$

Analogously to Proposition 1 in [5] one can prove

LEMma 3.7. Let $\Gamma_{v}$ be a closed fat proper cone in $\mathbb{R}^{n}$ and $b<_{\Gamma^{*}}$ a. Then $L_{b}^{\left(M_{p}\right)}\left(\Gamma_{v}\right)$ is contained in the closure of $Y_{a}$ in $L_{a}^{\left(M_{p}\right)}\left(\Gamma_{v}\right)$. Thus $Y_{(\omega)}$ is dense in $L_{(\omega)}^{\left(M_{p}\right)}\left(\Gamma_{v}\right)$.

4. The Seeley type theorem. In this section we show the extension type theorem for the space $L_{a}^{\left(M_{p}\right)}\left(\Gamma_{v}\right)$ of Laplace ultradifferentiable functions. For this purpose we prove the following lemmas

Lemma 4.1. Let $\Gamma \neq \mathbb{R}^{n}$ be a closed convex fat cone in $\mathbb{R}^{n}(n>1)$ with vertex at 0 . Fix $a>0$. Then there exists a linear continuous extension operator

$$
E_{1}: \mathcal{E}^{\left(M_{p}\right)}\left(\Gamma \cap\left\{x \in \mathbb{R}^{n}:\|x\| \geq a\right\}\right) \rightarrow \mathcal{E}^{\left(M_{p}\right)}\left(\mathbb{R}^{n}\right) .
$$

Proof. Let $\Phi: \mathbb{R}_{+} \times \mathbb{S}^{n-1} \rightarrow \mathbb{R}^{n}$ denote the spherical coordinate transformation. Since the ultradifferentiable functions of class $\left(M_{p}\right)$ are stable under real analytic coordinate transformations (see [4], p. 626), $\Phi$ gives the isomorphism of the space $\mathcal{E}^{\left(M_{p}\right)}(\Gamma \cap\{x \in$ 
$\left.\left.\mathbb{R}^{n}:\|x\| \geq a\right\}\right)$ onto $\mathcal{E}^{\left(M_{p}\right)}([a, \infty) \times S)$, where $S$ is a convex compact set in $\mathbb{R}^{n-1}$ satisfying $[a, \infty) \times S=\Phi^{-1}\left(\Gamma \cap\left\{x \in \mathbb{R}^{n}:\|x\| \geq a\right\}\right)$. So, it is sufficient to construct a linear continuous extension operator

$$
\tilde{E}_{1}: \mathcal{E}^{\left(M_{p}\right)}([a, \infty) \times S) \rightarrow \mathcal{E}^{\left(M_{p}\right)}\left(\mathbb{R}^{n}\right) .
$$

To this end, fix $\varepsilon \in(0, a / 2)$ and define a covering $\left\{U_{k}\right\}_{k \in \mathbb{N}_{0}}$ of $[a, \infty) \times S$ by the sets $U_{k}:=(a+k-\varepsilon, a+k+1+\varepsilon) \times(S)_{\varepsilon}$ for $k=1,2, \ldots$

Next, by Proposition 5.2 in [3], we may construct an ultradifferentiable locally finite partition of unity $\left\{\psi_{k}\right\}_{k \in \mathbb{N}_{0}}$ subordinate to $\left\{U_{k}\right\}_{k \in \mathbb{N}_{0}}$. Moreover in this partition of unity we can put $\psi_{k}(x):=\psi_{0}(x-(k, 0, \ldots, 0))$ for $k \in \mathbb{N}_{0}$. Hence the functions $\psi_{k}$ satisfy:

1) $\psi_{k} \in \mathcal{E}^{\left(M_{p}\right)}\left((a-\varepsilon, \infty) \times(S)_{\varepsilon}\right)$;

2) $\operatorname{supp} \psi_{k} \subset U_{k}$;

3) $\sum \psi_{k}(x)=1$ on $[a, \infty) \times S$;

4) the family $\left\{\psi_{k}\right\}_{k \in \mathbb{N}_{0}}$ is equibounded in $\mathcal{E}^{\left(M_{p}\right)}\left((a-\varepsilon, \infty) \times(S)_{\varepsilon}\right)$.

Furthermore, let $\tilde{E}_{1, k}$ (for $k \in \mathbb{N}_{0}$ ) be a linear continuous extension operator for ultradifferentiable functions on the convex compact set $\hat{U}_{k}:=[a+k-\varepsilon, a+k+1+\varepsilon] \times S$ (see Proposition 4.7 in [2]):

$$
\tilde{E}_{1, k}: \mathcal{E}^{\left(M_{p}\right)}\left(\hat{U}_{k}\right) \rightarrow \mathcal{E}^{\left(M_{p}\right)}\left(\mathbb{R}^{n}\right)
$$

such that:

1) $\operatorname{supp} \tilde{E}_{1, k}(\psi) \subset\left(\hat{U}_{k}\right)_{\varepsilon}$ for every $\psi \in \mathcal{E}^{\left(M_{p}\right)}\left(\hat{U}_{k}\right)$;

2) if $\psi \in \mathcal{E}^{\left(M_{p}\right)}\left(\hat{U}_{k}\right)$ and $\operatorname{supp} \psi \subset(a+k-\varepsilon, a+k+1+\varepsilon) \times S$ then $\operatorname{supp} \tilde{E}_{1, k}(\psi) \cap$ $([a, \infty) \times S)=\operatorname{supp} \psi$.

Since for every $k \in \mathbb{N}_{0}$ the set $\hat{U}_{k}$ is isometric to $\hat{U}_{1}$, we also can assume that:

3) the family $\left\{\tilde{E}_{1, k}\right\}_{k \in \mathbb{N}_{0}}$ of operators is equicontinuous.

Now, for every $\varphi \in \mathcal{E}^{\left(M_{p}\right)}([a-\varepsilon, \infty) \times S)$ one can define $\tilde{E}_{1}$ by

$$
\tilde{E}_{1}(\varphi):=\sum_{k \in \mathbb{N}_{0}} \tilde{E}_{1, k}\left(\psi_{k} \varphi\right)
$$

Observe that by the properties of the functions $\left\{\psi_{k}\right\}_{k \in \mathbb{N}_{0}}$ and the mappings $\left\{\tilde{E}_{1, k}\right\}_{k \in \mathbb{N}_{0}}$, $\tilde{E}_{1}$ is a linear continuous extension operator from $\mathcal{E}^{\left(M_{p}\right)}([a-\varepsilon, \infty) \times S)$ to $\mathcal{E}^{\left(M_{p}\right)}\left(\mathbb{R}^{n}\right)$. Hence in Cartesian coordinates we obtain a linear continuous extension operator

$$
E_{1}: \mathcal{E}^{\left(M_{p}\right)}\left(\Gamma \cap\left\{x \in \mathbb{R}^{n}:\|x\| \geq a\right\}\right) \rightarrow \mathcal{E}^{\left(M_{p}\right)}\left(\mathbb{R}^{n}\right),
$$

which completes the proof.

Lemma 4.2. Let $\Gamma$ be a closed convex fat cone in $\mathbb{R}^{n}(n>1)$ with vertex at 0 . Then there exists a linear continuous extension operator

$$
\tilde{E}: \mathcal{E}^{\left(M_{p}\right)}(\Gamma) \rightarrow \mathcal{E}^{\left(M_{p}\right)}\left(\mathbb{R}^{n}\right) .
$$

Proof. Fix $\varepsilon \in(0,1)$. By Proposition 5.2 in [3], there exists a partition of unity $\left\{\psi_{1}, \psi_{2}\right\}$ subordinate to $V_{1}:=\left(\Gamma \cap\left\{x \in \mathbb{R}^{n}:\|x\|>1\right\}\right)_{\varepsilon}, V_{2}:=\left(\Gamma \cap\left\{x \in \mathbb{R}^{n}:\|x\|<1\right\}\right)_{\varepsilon}$ satisfying: 
1) $\psi_{i} \in \mathcal{E}^{\left(M_{p}\right)}\left((\Gamma)_{\varepsilon}\right)$ for $i=1,2$

2) $\operatorname{supp} \psi_{i} \subset V_{i}$ for $i=1,2$;

3) $\psi_{1}(x)+\psi_{2}(x)=1$ on $\Gamma$.

Let $E_{1}: \mathcal{E}^{\left(M_{p}\right)}\left(\Gamma \cap \bar{V}_{1}\right) \rightarrow \mathcal{E}^{\left(M_{p}\right)}\left(\mathbb{R}^{n}\right)$ be a linear continuous extension operator constructed in Lemma 4.1

Moreover, by Proposition 4.7 in [2], we have also a linear continuous extension operator $E_{2}: \mathcal{E}^{\left(M_{p}\right)}\left(\Gamma \cap \bar{V}_{2}\right) \rightarrow \mathcal{E}^{\left(M_{p}\right)}\left(\mathbb{R}^{n}\right)$.

Obviously we may assume that if $\psi \in \mathcal{E}^{\left(M_{p}\right)}\left(\Gamma \cap \bar{V}_{i}\right)$ and $\operatorname{supp} \psi \subset \Gamma \cap V_{i}$ then $\operatorname{supp} E_{i}(\psi) \cap \Gamma=\operatorname{supp} \psi$ for $i=1,2$.

Now we can define $\tilde{E}$ by

$$
\tilde{E}(\varphi):=E_{1}\left(\psi_{1} \varphi\right)+E_{2}\left(\psi_{2} \varphi\right) \text { for every } \varphi \in \mathcal{E}^{\left(M_{p}\right)}(\Gamma) .
$$

Observe that $\tilde{E}: \mathcal{E}^{\left(M_{p}\right)}(\Gamma) \rightarrow \mathcal{E}^{\left(M_{p}\right)}\left(\mathbb{R}^{n}\right)$ is a continuous linear extension mapping.

TheOrem 4.3. Assume that $\Gamma_{v}$ is a closed convex fat cone in $\mathbb{R}^{n}$ with vertex at $v$. Then for every closed cone $\Gamma_{v^{\prime}}^{\prime} \subseteq \mathbb{R}^{n}$ satisfying $\Gamma_{v} \subset \operatorname{Int} \Gamma_{v^{\prime}}^{\prime}$ there exists a linear continuous extension operator

$$
E: L_{a}^{\left(M_{p}\right)}\left(\Gamma_{v}\right) \rightarrow L_{a}^{\left(M_{p}\right)}\left(\mathbb{R}^{n}\right)
$$

such that $\operatorname{supp} E(\varphi) \subset \Gamma_{v^{\prime}}^{\prime}$ for every $\varphi \in L_{a}^{\left(M_{p}\right)}\left(\Gamma_{v}\right)$.

Proof. Obviously in the case $\Gamma_{v}=\mathbb{R}^{n}$ it is sufficient to put $E:=\mathrm{Id}$. For $n=1$ the cone $\Gamma_{v}$ is an interval $[v, \infty)$ or $(-\infty, v]$ and the assertion is valid (see for instance [5] or [6]).

Hence we can assume that $n \geq 2$ and $\Gamma_{v} \neq \mathbb{R}^{n}$. Clearly, we can also put $v=0$. Applying Lemma 4.2 we see that there exists a linear continuous extension operator

$$
\tilde{E}: L_{0}^{\left(M_{p}\right)}\left(\Gamma_{v}\right) \rightarrow L_{0}^{\left(M_{p}\right)}\left(\mathbb{R}^{n}\right) .
$$

Since the operations of multiplication

$$
\exp _{a}: L_{0}^{\left(M_{p}\right)}\left(\mathbb{R}^{n}\right) \rightarrow L_{a}^{\left(M_{p}\right)}\left(\mathbb{R}^{n}\right) \text { and } \exp _{-a}: L_{a}^{\left(M_{p}\right)}\left(\Gamma_{v}\right) \rightarrow L_{0}^{\left(M_{p}\right)}\left(\Gamma_{v}\right)
$$

are linear and continuous isomorphisms, we define a linear continuous extension operator

$$
E^{\prime}: L_{a}^{\left(M_{p}\right)}\left(\Gamma_{v}\right) \rightarrow L_{a}^{\left(M_{p}\right)}\left(\mathbb{R}^{n}\right)
$$

by the formula

$$
E^{\prime}(\varphi):=\exp _{a} \cdot \tilde{E}\left(\exp _{-a} \cdot \varphi\right) \quad \text { for every } \varphi \in L_{a}^{\left(M_{p}\right)}\left(\Gamma_{v}\right) .
$$

By Proposition 5.2 in [3], there exists a cut-off function $\chi_{\Gamma_{v}, \Gamma_{v^{\prime}}^{\prime}} \in L_{0}^{\left(M_{p}\right)}\left(\mathbb{R}^{n}\right)$ such that $0 \leq \chi_{\Gamma_{v}, \Gamma_{v^{\prime}}^{\prime}}(x) \leq 1$ for $x \in \mathbb{R}^{n}, \chi_{\Gamma_{v}, \Gamma_{v^{\prime}}}(x)=1$ for $x \in \Gamma_{v}^{v^{\prime}}$ and $\chi_{\Gamma_{v}, \Gamma_{v^{\prime}}^{\prime}}(x)=0$ for $x \in \mathbb{R}^{n} \backslash \Gamma_{v^{\prime}}^{\prime \prime}$. Since the multiplication by $\chi_{\Gamma_{v}, \Gamma_{v^{\prime}}^{\prime}}$ is a linear continuous isomorphism in $L_{a}^{\left(M_{p}\right)}\left(\mathbb{R}^{n}\right)$, we can construct a linear continuous extension operator

$$
E: L_{a}^{\left(M_{p}\right)}\left(\Gamma_{v}\right) \rightarrow L_{a}^{\left(M_{p}\right)}\left(\mathbb{R}^{n}\right)
$$

as

$$
E(\varphi):=\chi_{\Gamma_{v}, \Gamma_{v^{\prime}}^{\prime}} \cdot E^{\prime}(\varphi) \text { for every } \varphi \in L_{a}^{\left(M_{p}\right)}\left(\Gamma_{v}\right) .
$$

Clearly supp $E(\varphi) \subset \Gamma_{v^{\prime}}^{\prime}$ for every $\varphi \in L_{a}^{\left(M_{p}\right)}\left(\Gamma_{v}\right)$. 
Using the extension mapping $E$ constructed in Theorem 4.3 and following the proof of Proposition 5.1 in [8] we obtain

Corollary 4.4. Let $\Gamma_{v}$ be a closed convex fat cone in $\mathbb{R}^{n}$. Then the space $L_{a}^{\left(M_{p}\right)}\left(\Gamma_{v}\right)$ is complete.

5. The Paley-Wiener-Schwartz type theorem. In this section we shall prove that the Laplace transform defines an isomorphism between the space $L_{(\omega)}^{\prime\left(M_{p}\right)}\left(\Gamma_{v}\right)$ of Laplace ultradistributions and some space of holomorphic functions with given growth condition. Definition 5.1. The Laplace transform of $u \in L_{(\omega)}^{\prime\left(M_{p}\right)}\left(\Gamma_{v}\right)$ is a function defined by

$$
\mathcal{L} u(z):=u\left[\exp _{z}\right] \text { for } \operatorname{Re} z<_{\Gamma^{*}} \omega \text {. }
$$

Observe that by Example 3.5 the Laplace transform is well defined.

THEOREM 5.2. Let $\Gamma_{v}$ be a closed fat proper cone in $\mathbb{R}^{n}$ with vertex at $v, u \in L_{(\omega)}^{\prime\left(M_{p}\right)}\left(\Gamma_{v}\right)$ and $F(z):=\mathcal{L} u(z)$ for $\operatorname{Re} z<_{\Gamma^{*}} \omega$. Then $F$ is a holomorphic function on $\operatorname{Re} z<_{\Gamma^{*}} \omega$ and for every $a<_{\Gamma^{*}} \omega$ there exist $h(a)>0$ and $C(a)<\infty$ such that

$$
|F(z)| \leq C(a) e^{v \cdot \operatorname{Re} z} \exp M\left(\frac{\left|z_{1}\right|}{h(a)}\right) \cdots \exp M\left(\frac{\left|z_{n}\right|}{h(a)}\right) \quad \text { for } \operatorname{Re} z<_{\Gamma^{*}} a .
$$

Proof. Since $u$ is a linear functional on $L_{(\omega)}^{\left(M_{p}\right)}\left(\Gamma_{v}\right)$ and the mapping $z \mapsto \exp _{z}$ is holomorphic on $\left\{\operatorname{Re} z<_{\Gamma^{*}} \omega\right\}$, it follows that $F(z)$ satisfies our assertion.

THEOREM 5.3. Let $\Gamma_{v}$ be a closed fat proper cone in $\mathbb{R}^{n}, \omega_{1}, \omega_{2} \in(\mathbb{R} \cup\{\infty\})^{n}$ satisfies $\omega_{1} \leq_{\Gamma^{*}} \omega_{2}, u_{1} \in L_{\left(\omega_{1}\right)}^{\prime\left(M_{p}\right)}\left(\Gamma_{v}\right), u_{2} \in L_{\left(\omega_{2}\right)}^{\prime\left(M_{p}\right)}\left(\Gamma_{v}\right)$. If $\mathcal{L} u_{1}(z)=\mathcal{L} u_{2}(z)$ for $\operatorname{Re} z<_{\Gamma^{*}} \omega_{1}$ then $u_{1}=u_{2}$ in $L_{\left(\omega_{1}\right)}^{\prime\left(M_{p}\right)}\left(\Gamma_{v}\right)$.

Proof. Fix $\varphi \in L_{\left(\omega_{1}\right)}^{\left(M_{p}\right)}\left(\Gamma_{v}\right)$. It is sufficient to show that $u_{1}[\varphi]=u_{2}[\varphi]$. For this purpose choose $b<_{\Gamma^{*}} \omega_{1}$ such that $\varphi \in L_{b}^{\left(M_{p}\right)}\left(\Gamma_{v}\right)$ and take $b<_{\Gamma^{*}} a<_{\Gamma^{*}} \omega_{1}$. By the assumption $u_{1}\left[\exp _{c}\right]=u_{2}\left[\exp _{c}\right]$ for every $c \leq_{\Gamma^{*}} a$. Hence the proof follows from Lemma 3.7 .

To prove the converse of Theorem 5.2 we need a few lemmas. The first one is a version of Lemma 9.1 in [8] (see also Lemma 3.7 in [5]).

Lemma 5.4. Let $\Gamma_{v}$ be a closed fat proper convex cone in $\mathbb{R}^{n}$ with vertex at $v$. Suppose that $a \in \mathbb{R}^{n}$ and $G$ is a holomorphic function on the set $\left\{z \in \mathbb{C}^{n}: \operatorname{Re} z \leq_{\Gamma^{*}}\right.$ a $\}$ satisfying the estimation

$$
|G(z)| \leq \frac{C}{\left\langle z_{1}\right\rangle^{2} \cdots\left\langle z_{n}\right\rangle^{2}} e^{v \cdot \operatorname{Re} z} \quad \text { for some } C<\infty .
$$

Then

$$
g(y):=(2 \pi i)^{-n} \int_{c+i \mathbb{R}^{n}} G(z) e^{-y \cdot z} d z \quad \text { for } y \in \mathbb{R}^{n}
$$

defines correctly a function $g$ such that:

a) $g$ does not depend on the choice of $c \leq_{\Gamma^{*}}$ a;

b) $g$ is a continuous function on $\mathbb{R}^{n}$;

c) $\operatorname{supp} g \subset \Gamma_{v}$;

d) the function $\Gamma_{v} \ni y \mapsto e^{a \cdot y} g(y)$ is bounded; 
e) $g \in L_{(a)}^{\prime\left(M_{p}\right)}\left(\Gamma_{v}\right)$;

f) $G(z)=\mathcal{L} g(z)$ for $\operatorname{Re} z<_{\Gamma^{*}} a$.

Proof. See the proof of Lemma 9.1 in [8].

Lemma 5.5. Let $\Gamma$ be a convex proper cone in $\mathbb{R}^{n}$ with vertex at 0 . Then there exists a linear transformation $\Phi: \mathbb{R}^{n} \rightarrow \mathbb{R}^{n}$ satisfying the following conditions:

1) $\Phi(\Gamma) \subset \overline{\mathbb{R}}_{+}^{n}$,

2) $\|x\| \leq\|\Phi(x)\|$ for every $x \in \mathbb{R}^{n}$.

Proof. Since $\Gamma$ is a proper cone with vertex at 0 , we can find $A \in \mathrm{SO}(n, \mathbb{R})$ such that

$$
A(\Gamma) \subset \overline{\mathbb{R}}_{+} \times \mathbb{R}^{n-1} \text { and } A(\Gamma) \cap\left(\{0\} \times \mathbb{R}^{n-1}\right)=\{0\} .
$$

Next, we can choose a sufficiently large $\lambda \geq 1$ such that for the linear operator $S: \mathbb{R}^{n} \rightarrow \mathbb{R}^{n}$ defined by

$$
S\left(x_{1}, \ldots, x_{n}\right):=\left(\lambda x_{1}, x_{2}, \ldots, x_{n}\right)
$$

there exists $T \in \mathrm{SO}(n, \mathbb{R})$ satisfying $T(S(A(\Gamma))) \subset \overline{\mathbb{R}}_{+}^{n}$.

To finish the proof, it is sufficient to put $\Phi(x):=T(S(A(x)))$.

Lemma 5.6. Let $\Phi: \mathbb{R}^{n} \rightarrow \mathbb{R}^{n}$ be the linear transformation constructed in the previous lemma. If $\hat{\Phi}: \mathbb{C}^{n} \rightarrow \mathbb{C}^{n}$ is the complexification of $\Phi($ i.e. $\hat{\Phi}(x+i y):=\Phi(x)+i \Phi(y))$ then

$$
\begin{gathered}
\|z\| \leq\|\hat{\Phi}(z)\|, \\
\left|z_{i}\right| \leq \sqrt{n} \max _{j=1, \ldots, n}\left|\hat{\Phi}_{j}(z)\right| \quad \text { for } i=1, \ldots, n, \\
\left\langle z_{1}\right\rangle \cdots\left\langle z_{n}\right\rangle \leq \sqrt{n}^{n}\left\langle\hat{\Phi}_{1}(z)\right\rangle^{n} \cdots\left\langle\hat{\Phi}_{n}(z)\right\rangle^{n} .
\end{gathered}
$$

Proof. By Lemma 5.5

$$
\|z\|^{2}=\|x\|^{2}+\|y\|^{2} \leq\|\Phi(x)\|^{2}+\|\Phi(y)\|^{2}=\|\hat{\Phi}(z)\|^{2}
$$

and (1) holds. Hence

$$
\left|z_{i}\right|^{2} \leq\|z\|^{2} \leq\|\hat{\Phi}(z)\|^{2} \leq n \max _{j=1, \ldots, n}\left|\hat{\Phi}_{j}(z)\right|^{2} \quad \text { for } \quad i=1, \ldots, n,
$$

which gives (2). To prove (3) observe that by (2)

$$
\left\langle z_{i}\right\rangle=1+\left|z_{i}\right| \leq 1+\sqrt{n}\left(\max _{j=1, \ldots, n}\left|\hat{\Phi}_{j}(z)\right|\right) \leq \sqrt{n} \max _{j=1, \ldots, n}\left\langle\hat{\Phi}_{j}(z)\right\rangle \leq \sqrt{n}\left\langle\hat{\Phi}_{1}(z)\right\rangle \cdots\left\langle\hat{\Phi}_{n}(z)\right\rangle .
$$

Thus

$$
\left\langle z_{1}\right\rangle \cdots\left\langle z_{n}\right\rangle \leq \sqrt{n}^{n}\left\langle\hat{\Phi}_{1}(z)\right\rangle^{n} \cdots\left\langle\hat{\Phi}_{n}(z)\right\rangle^{n},
$$

which completes the proof.

Lemma 5.7. Let $\Gamma_{v}$ be a proper cone in $\mathbb{R}^{n}, \omega \in \mathbb{R}^{n}$ and $k>0$. Then there exists a symbol $P$ of class $\left(M_{p}\right)$ non-vanishing on $\left\{z \in \mathbb{C}^{n}: \operatorname{Re} z \leq_{\Gamma^{*}} \omega\right\}$ and such that

$$
\frac{\exp M\left(k\left|z_{1}\right|\right) \cdots \exp M\left(k\left|z_{n}\right|\right)}{|P(z)|} \leq \frac{C}{\left\langle z_{1}\right\rangle^{2} \cdots\left\langle z_{n}\right\rangle^{2}} \quad \text { for } \quad \operatorname{Re} z<_{\Gamma^{*}} \omega \text {. }
$$


Proof. Let $\hat{\Phi}(z)$ be the complexification of the linear transformation with respect to $\Gamma^{*}$ constructed in Lemma 5.5 . By 2

$$
\begin{aligned}
\exp M\left(k\left|z_{i}\right|\right) & \leq \exp M\left(k \sqrt{n} \max _{j=1, \ldots, n}\left|\hat{\Phi}_{j}(z)\right|\right) \\
& \leq \exp M\left(k \sqrt{n}\left|\hat{\Phi}_{1}(z)\right|\right) \cdots \exp M\left(k \sqrt{n}\left|\hat{\Phi}_{n}(z)\right|\right) .
\end{aligned}
$$

This gives

$$
\exp M\left(k\left|z_{1}\right|\right) \cdots \exp M\left(k\left|z_{n}\right|\right) \leq\left(\exp M\left(k \sqrt{n}\left|\hat{\Phi}_{1}(z)\right|\right) \cdots \exp M\left(k \sqrt{n}\left|\hat{\Phi}_{n}(z)\right|\right)\right)^{n} .
$$

Since $m_{p} \rightarrow \infty$ as $p \rightarrow \infty$, we can choose $p_{0} \in \mathbb{N}$ such that

$$
m_{p}>2 k\left|\hat{\Phi}_{i}(\omega)\right|+k \quad \text { and } \quad\left|m_{p}-k \hat{\Phi}_{i}(z)\right| \geq k\left|\hat{\Phi}_{i}(z)\right|
$$

for $i=1, \ldots, n, p \geq p_{0}$ and $\operatorname{Re} z<_{\Gamma^{*}} \omega$.

Fix $i \in\{1, \ldots, n\}$. By Lemma 5.5

$$
\operatorname{Re} \hat{\Phi}_{i}(z)=\Phi_{i}(\operatorname{Re} z) \leq \Phi_{i}(\omega) \text { for } \operatorname{Re} z \leq_{\Gamma^{*}} \omega .
$$

Let

$$
P_{i}(z):=\left(\hat{\Phi}_{i}(\omega-z)+1\right)^{p_{0}+1} \prod_{p=p_{0}}^{\infty}\left(1-\frac{k \sqrt{n} \hat{\Phi}_{i}(z)}{m_{p}}\right) \quad \text { for } \quad z \in \mathbb{C}^{n}
$$

Then $P_{i}(z)$ does not vanish on $\left\{z \in \mathbb{C}^{n}: \operatorname{Re} z \leq_{\Gamma^{*}} \omega\right\}$. On the other hand, by the Hadamard factorisation theorem (Propositions 4.5 and 4.6 in [3]), $P_{i}(z)$ is a symbol of class $\left(M_{p}\right)$. Moreover, it satisfies the inequality (see Lemma 3 in [5])

$$
\frac{\exp M\left(k \sqrt{n}\left|\hat{\Phi}_{i}(z)\right|\right)}{\left|P_{i}(z)\right|} \leq \frac{C_{i}}{\left\langle\hat{\Phi}_{i}(z)\right\rangle^{2}} \quad \text { for } \quad \operatorname{Re} z \leq_{\Gamma^{*}} \omega
$$

with some $C_{i}<\infty$.

Taking $P(z):=P_{1}(z)^{n} \cdots P_{n}(z)^{n}$ we see that $P(z)$ is also a symbol of class $\left(M_{p}\right)$ non-vanishing on $\left\{z \in \mathbb{C}^{n}: \operatorname{Re} z \leq_{\Gamma^{*}} \omega\right\}$. Furthermore, by (3)-(5) we have

$$
\begin{aligned}
\frac{\exp M\left(k\left|z_{1}\right|\right) \cdots \exp M\left(k\left|z_{n}\right|\right)}{|P(z)|} & \leq\left(\frac{\exp M\left(k \sqrt{n}\left|\hat{\Phi}_{1}(z)\right|\right) \cdots \exp M\left(k \sqrt{n}\left|\hat{\Phi}_{n}(z)\right|\right)}{\left|P_{1}(z)\right| \cdots\left|P_{n}(z)\right|}\right)^{n} \\
& \leq \frac{C_{1}^{n} \cdots C_{n}^{n}}{\left\langle\hat{\Phi}_{1}(z)\right\rangle^{2 n} \cdots\left\langle\hat{\Phi}_{n}(z)\right\rangle^{2 n}} \leq \frac{n^{n} C_{1}^{n} \cdots C_{n}^{n}}{\left\langle z_{1}\right\rangle^{2} \cdots\left\langle z_{n}\right\rangle^{2}}
\end{aligned}
$$

for $\operatorname{Re} z<_{\Gamma^{*}} \omega$.

THEOREM 5.8. Let $\omega \in \mathbb{R}^{n}$ and $\Gamma_{v}$ be a closed convex fat proper cone in $\mathbb{R}^{n}$ with vertex at $v \in \mathbb{R}^{n}$. Moreover, let $F$ be a function holomorphic on $\left\{\operatorname{Re} z<_{\Gamma^{*}} \omega\right\}$ and such that for every $a<_{\Gamma^{*}} \omega$ there exist $h(a)>0$ and $C(a)<\infty$ satisfying

$$
|F(z)| \leq C(a) e^{v \cdot \operatorname{Re} z} \exp M\left(\frac{\left|z_{1}\right|}{h(a)}\right) \cdots \exp M\left(\frac{\left|z_{n}\right|}{h(a)}\right) \quad \text { for } \quad \operatorname{Re} z<_{\Gamma^{*}} a .
$$

Then there exists exactly a unique Laplace ultradistribution $S \in L_{(\omega)}^{\prime\left(M_{p}\right)}\left(\Gamma_{v}\right)$ satisfying

$$
F(z)=\mathcal{L} S(z) \quad \text { for } \quad \operatorname{Re} z<_{\Gamma^{*}} \omega .
$$


Proof. Fix $a<_{\Gamma^{*}} \omega$ and choose $\tilde{\omega} \in \mathbb{R}^{n}$ such that $a<_{\Gamma^{*}} \tilde{\omega}<_{\Gamma^{*}} \omega$. By Lemma 5.7 there exists a symbol $P(z)$ of class $\left(M_{p}\right)$ such that

$$
\left|\frac{F(z)}{P(z)}\right| \leq \frac{C e^{v \cdot \operatorname{Re} z}}{\left\langle z_{1}\right\rangle^{2} \cdots\left\langle z_{n}\right\rangle^{2}} \quad \text { for } \quad \operatorname{Re} z \leq_{\Gamma^{*}} \tilde{\omega} .
$$

Put $G(z):=\frac{F(z)}{P(z)}$. By Lemma 5.4 there exists $g \in L_{(a)}^{\prime\left(M_{p}\right)}\left(\Gamma_{v}\right)$ satisfying $\mathcal{L} g(z)=G(z)$ for $\operatorname{Re} z<_{\Gamma^{*}} a$.

Let $S_{a}:=P(-D) g(z)$. Then $S_{a} \in L_{(a)}^{\prime\left(M_{p}\right)}\left(\Gamma_{v}\right)$ and $\mathcal{L} S_{a}(z)=P(z) \mathcal{L} g(z)=P(z) G(z)=$ $F(z)$ for $\operatorname{Re} z<_{\Gamma^{*}} a$. The formula $S=S_{a}$ on $L_{(a)}^{\left(M_{p}\right)}\left(\Gamma_{v}\right)$ for every $a<_{\Gamma^{*}} \omega$ defines correctly a functional $S \in L_{(\omega)}^{\prime\left(M_{p}\right)}\left(\Gamma_{v}\right)$, which satisfies 6 .

Combining Theorems 5.2, 5.3 and 5.8 we obtain

Theorem 5.9 (Paley-Wiener-Schwartz type theorem). Let $\Gamma_{v}$ be a closed convex fat proper cone in $\mathbb{R}^{n}$ with vertex at $v$. Then a function $F(z)$ is the Laplace transform of the unique Laplace ultradistribution $u \in L_{(\omega)}^{\prime\left(M_{p}\right)}\left(\Gamma_{v}\right)$ if and only if $F(z)$ is a holomorphic function on the set $\left\{\operatorname{Re} z<_{\Gamma^{*}} \omega\right\}$ and for every $a<_{\Gamma^{*}} \omega$ there exist $h(a)>0$ and $C(a)<\infty$ such that

$$
|F(z)| \leq C(a) e^{v \cdot \operatorname{Re} z} \exp M\left(\frac{\left|z_{1}\right|}{h(a)}\right) \cdots \exp M\left(\frac{\left|z_{n}\right|}{h(a)}\right) \quad \text { for } \quad \operatorname{Re} z<_{\Gamma^{*}} a .
$$

By the proof of Theorem 5.8 we obtain the characterisation of Laplace ultradistributions supported by a cone. In this way we extend the result of G. Łysik (see Theorem 5 in [5]) to the proper convex cone.

THEOREM 5.10 (Structure theorem). Let $\Gamma_{v}$ be a closed convex fat proper cone in $\mathbb{R}^{n}$ with vertex at $v$. Then an ultradistribution $S \in D^{\prime\left(M_{p}\right)}\left(\mathbb{R}^{n}\right)$ belongs to the space $L_{(\omega)}^{\left(M_{p}\right)}\left(\Gamma_{v}\right)$ if and only if for every $a<_{\Gamma^{*}} \omega$ there exist an ultradifferentiable operator $P_{a}$ of class $\left(M_{p}\right)$ and a continuous function $g_{a}$ on $\mathbb{R}^{n}$ supported by $\Gamma_{v}$ and satisfying:
a) $\left|g_{a}(y)\right| \leq C e^{-a \cdot y} \quad$ for $\quad y \in \Gamma_{v}$;
b) $\left|\mathcal{L} g_{a}(z)\right| \leq \frac{C}{\left\langle z_{1}\right\rangle^{2} \cdots\left\langle z_{n}\right\rangle^{2}}$ for $\operatorname{Re} z<_{\Gamma^{*}} a$;
c) $S=P_{a}(D) g_{a} \quad$ in $\quad L_{(a)}^{\prime\left(M_{p}\right)}\left(\Gamma_{v}\right)$.

6. The kernel theorem. In the last section we shall prove the kernel theorem for Laplace ultradistributions $L_{(\omega)}^{\prime\left(M_{p}\right)}\left(\Gamma_{v}\right)$. This version of the kernel theorem is a generalisation of Theorem 1 in [6] to the closed convex fat proper cones.

Definition 6.1. Let $v_{1} \in \mathbb{R}^{n_{1}}, v_{2} \in \mathbb{R}^{n_{2}}, \omega_{1} \in(\mathbb{R} \cup\{\infty\})^{n_{1}}, \omega_{2} \in(\mathbb{R} \cup\{\infty\})^{n_{2}}$. Moreover we assume that $\Gamma_{v_{1}}^{1} \subset \mathbb{R}^{n_{1}}$ and $\Gamma_{v_{2}}^{2} \subset \mathbb{R}^{n_{2}}$ are proper closed fat convex cones.

We denote by $L_{\left(\omega_{1}\right)}^{\prime\left(M_{p}\right)}\left(\Gamma_{v_{1}}^{1}, L_{\left(\omega_{2}\right)}^{\prime\left(M_{p}\right)}\left(\Gamma_{v_{2}}^{2}\right)\right)$ the space of Laplace ultradistributions on $\Gamma_{v_{1}}^{1}$ with values in $L_{\left(\omega_{2}\right)}^{\prime\left(M_{p}\right)}\left(\Gamma_{v_{2}}^{2}\right)$, i.e.

$$
A \in L_{\left(\omega_{1}\right)}^{\prime\left(M_{p}\right)}\left(\Gamma_{v_{1}}^{1}, L_{\left(\omega_{2}\right)}^{\prime\left(M_{p}\right)}\left(\Gamma_{v_{2}}^{2}\right)\right)
$$


if for any $\varphi \in L_{\left(\omega_{1}\right)}^{\left(M_{p}\right)}\left(\Gamma_{v_{1}}^{1}\right)$ we have $A[\varphi] \in L_{\left(\omega_{2}\right)}^{\prime\left(M_{p}\right)}\left(\Gamma_{v_{2}}^{2}\right)$ and the mapping

$$
L_{\left(\omega_{1}\right)}^{\left(M_{p}\right)}\left(\Gamma_{v_{1}}^{1}\right) \ni \varphi \mapsto A[\varphi] \in L_{\left(\omega_{2}\right)}^{\prime\left(M_{p}\right)}\left(\Gamma_{v_{2}}^{2}\right)
$$

is linear and continuous.

TheOREM 6.2 (The kernel theorem). The mapping

$$
\mathcal{I}_{M_{p}}: L_{\left(\omega_{1}, \omega_{2}\right)}^{\prime\left(M_{p}\right)}\left(\Gamma_{v_{1}}^{1} \times \Gamma_{v_{2}}^{2}\right) \rightarrow L_{\left(\omega_{1}\right)}^{\prime\left(M_{p}\right)}\left(\Gamma_{v_{1}}^{1}, L_{\left(\omega_{2}\right)}^{\prime\left(M_{p}\right)}\left(\Gamma_{v_{2}}^{2}\right)\right)
$$

such that for any $\tilde{A} \in L_{\left(\omega_{1}, \omega_{2}\right)}^{\prime\left(M_{p}\right)}\left(\Gamma_{v_{1}}^{1} \times \Gamma_{v_{2}}^{2}\right)$

$$
\mathcal{I}_{M_{p}}(\widetilde{A})[\varphi][\psi]:=\widetilde{A}[\varphi \otimes \psi] \quad \text { for } \quad \varphi \in L_{\left(\omega_{1}\right)}^{\left(M_{p}\right)}\left(\Gamma_{v_{1}}^{1}\right), \psi \in L_{\left(\omega_{2}\right)}^{\left(M_{p}\right)}\left(\Gamma_{v_{2}}^{2}\right),
$$

where $\varphi \otimes \psi$ denotes the function $\Gamma_{v_{1}}^{1} \times \Gamma_{v_{2}}^{2} \ni(x, y) \mapsto \varphi(x) \psi(y)$, is a linear topological isomorphism of $L_{\left(\omega_{1}, \omega_{2}\right)}^{\prime\left(M_{p}\right)}\left(\Gamma_{v_{1}}^{1} \times \Gamma_{v_{2}}^{2}\right)$ onto $L_{\left(\omega_{1}\right)}^{\prime\left(M_{p}\right)}\left(\Gamma_{v_{1}}^{1}, L_{\left(\omega_{2}\right)}^{\prime\left(M_{p}\right)}\left(\Gamma_{v_{2}}^{2}\right)\right)$.

Proof. Following the proof of Theorem 1 in [6], we can show that the operator $\mathcal{I}_{M_{p}}$ is well defined and continuous.

Next we construct a continuous inverse operator

$$
\mathcal{I}_{M_{p}}^{-1}: L_{\left(\omega_{1}\right)}^{\prime\left(M_{p}\right)}\left(\Gamma_{v_{1}}^{1}, L_{\left(\omega_{2}\right)}^{\prime\left(M_{p}\right)}\left(\Gamma_{v_{2}}^{2}\right)\right) \rightarrow L_{\left(\omega_{1}, \omega_{2}\right)}^{\prime\left(M_{p}\right)}\left(\Gamma_{v_{1}}^{1} \times \Gamma_{v_{2}}^{2}\right)
$$

such that

$$
\mathcal{I}_{M_{p}} \mathcal{I}_{M_{p}}^{-1}=\operatorname{Id}_{L_{\left(\omega_{1}\right)}^{\prime\left(M_{p}\right)}\left(\Gamma_{v_{1}}^{1}, L_{\left(\omega_{2}\right)}^{\prime\left(M_{p}\right)}\left(\Gamma_{v_{2}}^{2}\right)\right)} \quad \text { and } \quad \mathcal{I}_{M_{p}}^{-1} \mathcal{I}_{M_{p}}=\operatorname{Id}_{L_{\left(\omega_{1}, \omega_{2}\right)}^{\prime\left(M_{p}\right)}\left(\Gamma_{v_{1}}^{1} \times \Gamma_{v_{2}}^{2}\right)} .
$$

To this end fix $A \in L_{\left(\omega_{1}\right)}^{\prime\left(M_{p}\right)}\left(\Gamma_{v_{1}}^{1}, L_{\left(\omega_{2}\right)}^{\prime\left(M_{p}\right)}\left(\Gamma_{v_{2}}^{2}\right)\right)$ and take any $a_{1}, a_{2}, d_{1}, d_{2}$ such that $a_{j}<_{\Gamma^{j *}} d_{j}<_{\Gamma^{j *}} \omega_{j}$ (for $j=1,2$ ). By Corollary 4.4 and the Mazur-Orlicz theorem (see Theorem 2 in [6]) there exist $c_{A}<\infty$ and $h>0$ such that

$$
|A[\varphi][\psi]| \leq c_{A}\|\varphi\|_{\Gamma_{1}, d_{1}, h}^{\left(M_{p}\right)}\|\psi\|_{\Gamma_{2}, d_{2}, h}^{\left(M_{p}\right)} \quad \text { for } \quad \varphi \in L_{d_{1}}^{\left(M_{p}\right)}\left(\Gamma_{1}\right), \psi \in L_{d_{2}}^{\left(M_{p}\right)}\left(\Gamma_{2}\right) .
$$

By the Hahn-Banach theorem (7) holds for $\varphi \in L_{d_{1}, h}^{\left(M_{p}\right)}\left(\Gamma_{1}\right)$ and $\psi \in L_{d_{2}, h}^{\left(M_{p}\right)}\left(\Gamma_{2}\right)$. Put $\zeta_{j}:=b_{j}+i \eta_{j}$ where $b_{j} \in \mathbb{R}^{n_{j}}$, with $a_{j}<_{\Gamma^{j *}} b_{j}<_{\Gamma}{ }^{j *} d_{j}$ and $\eta_{j}=\left(\eta_{j 1}, \ldots, \eta_{j n_{j}}\right) \in \mathbb{R}^{n_{j}}$ for $j=1,2$. Since there exist $c_{j}, k_{j}\left(k_{j}:=\frac{\left\langle b_{j}\right\rangle}{h}\right)$ such that

$$
\left\|\exp _{\zeta_{j}}\right\|_{\Gamma_{j}, d_{j}, h,}^{\left(M_{p}\right)} \leq c_{j} \prod_{i=1}^{n_{j}} \exp M\left(k_{j}\left\langle\eta_{j i}\right\rangle\right)
$$

the function $\Gamma_{j} \ni x_{j} \mapsto \exp _{\zeta_{j}} x_{j}$ belongs to $L_{d_{j}, h}^{\left(M_{p}\right)}\left(\Gamma_{j}\right)$ (for $j=1,2$ ). So we conclude from (7) that

$$
\left|A\left[\exp _{\zeta_{1}}\right]\left[\exp _{\zeta_{2}}\right]\right| \leq c_{A} c_{1} c_{2} \prod_{i=1}^{n_{1}} \exp M\left(k_{1}\left\langle\eta_{1 i}\right\rangle\right) \prod_{i=1}^{n_{2}} \exp M\left(k_{2}\left\langle\eta_{2 i}\right\rangle\right)
$$

Let $\Phi \in L_{a_{1}, a_{2}}^{\left(M_{p}\right)}\left(\Gamma_{1} \times \Gamma_{2}\right)$. Then the Laplace transform $\mathcal{L} \Phi$ given by

$$
\mathcal{L} \Phi\left(\zeta_{1}, \zeta_{2}\right):=\int_{\Gamma_{1} \times \Gamma_{2}} \Phi\left(x_{1}, x_{2}\right) e^{-\zeta_{1} \cdot x_{1}-\zeta_{2} \cdot x_{2}} d x_{1} d x_{2} \quad \text { for } \quad a_{j}<_{\Gamma^{j *}} \operatorname{Re} \zeta_{j} \quad(j=1,2)
$$

satisfies

$$
\left|\mathcal{L} \Phi\left(\zeta_{1}, \zeta_{2}\right)\right| \leq c\|\Phi\|_{\Gamma_{1} \times \Gamma_{2},\left(a_{1}, a_{2}\right), 1}^{\left(M_{p}\right)}=: c_{\Phi}<\infty
$$


Put $P\left(\zeta_{1}, \zeta_{2}\right):=P^{(1)}\left(\zeta_{1}\right) P^{(2)}\left(\zeta_{2}\right)$, where $P^{(j)}\left(\zeta_{j}\right)$ is a symbol of class $\left(M_{p}\right)$ non-vanishing on $\left\{z \in \mathbb{C}^{n_{j}}: \operatorname{Re} z \leq_{\Gamma^{j *}} \omega_{j}\right\}$ and constructed in Lemma 5.7 (for $j=1,2$ ). Hence $P\left(\zeta_{1}, \zeta_{2}\right)$ is also a symbol of class $\left(M_{p}\right)$ and it satisfies the inequality (see Lemma 3 in [5])

$$
\begin{aligned}
& \frac{\exp M\left(k_{1}\left|\zeta_{11}\right|\right) \cdots \exp M\left(k_{1}\left|\zeta_{1 n_{1}}\right|\right) \exp M\left(k_{2}\left|\zeta_{21}\right|\right) \cdots \exp M\left(k_{2}\left|\zeta_{2 n_{2}}\right|\right)}{\left|P\left(\zeta_{1}, \zeta_{2}\right)\right|} \\
& \leq \frac{K^{\prime}}{\left\langle\eta_{11}\right\rangle^{2} \cdots\left\langle\eta_{1 n_{1}}\right\rangle^{2}\left\langle\eta_{21}\right\rangle^{2} \cdots\left\langle\eta_{2 n_{2}}\right\rangle^{2}}
\end{aligned}
$$

with some $K^{\prime}<\infty$.

Now we can define the mapping $\mathcal{I}_{M_{p}}^{-1}$ by

$$
\mathcal{I}_{M_{p}}^{-1}(A)[\Phi]:=\left(\frac{1}{2 \pi i}\right)^{n_{1}+n_{2}} P\left(D_{x_{1}}, D_{x_{2}}\right) \int_{b_{1}+i \mathbb{R}^{n_{1}}} \int_{b_{2}+i \mathbb{R}^{n_{2}}} A\left[\exp _{\zeta_{1}}\right]\left[\exp _{\zeta_{2}}\right] \frac{\mathcal{L} \Phi\left(\zeta_{1}, \zeta_{2}\right)}{P\left(\zeta_{1}, \zeta_{2}\right)} d \zeta_{1} d \zeta_{2} .
$$

From (8)-10 we obtain

$$
\begin{aligned}
& \left|A\left[\exp _{\zeta_{1}}\right]\left[\exp _{\zeta_{2}}\right] \frac{\mathcal{L} \Phi\left(\zeta_{1}, \zeta_{2}\right)}{P\left(\zeta_{1}, \zeta_{2}\right)}\right| \\
& \leq c_{A} c_{1} c_{2} c_{\Phi} \frac{\exp M\left(k_{1}\left\langle\eta_{11}\right\rangle\right) \cdots \exp M\left(k_{1}\left\langle\eta_{1 n_{1}}\right\rangle\right) \exp M\left(k_{2}\left\langle\eta_{21}\right\rangle\right) \cdots \exp M\left(k_{2}\left\langle\eta_{2 n_{2}}\right\rangle\right)}{\left|P\left(\zeta_{1}, \zeta_{2}\right)\right|} \\
& \leq \frac{K}{\left\langle\eta_{11}\right\rangle^{2} \cdots\left\langle\eta_{1 n_{1}}\right\rangle^{2}\left\langle\eta_{21}\right\rangle^{2} \cdots\left\langle\eta_{2 n_{2}}\right\rangle^{2}}
\end{aligned}
$$

with some $K<\infty$. Therefore the integral in 11 is convergent.

Since the ultradifferential operator

$$
P\left(D_{x_{1}}, D_{x_{2}}\right): L_{a_{1}, a_{2}}^{\left(M_{p}\right)}\left(\Gamma_{1} \times \Gamma_{2}\right) \rightarrow L_{a_{1}, a_{2}}^{\left(M_{p}\right)}\left(\Gamma_{1} \times \Gamma_{2}\right)
$$

is continuous (see Theorem 2.12 in [3]), we have for $h>0$ sufficiently small

$$
\left|\mathcal{I}_{M_{p}}^{-1}(A)[\Phi]\right| \leq C c_{A}\|\Phi\|_{\Gamma_{1} \times \Gamma_{2},\left(a_{1}, a_{2}\right), h}^{\left(M_{p}\right)}
$$

with some $C<\infty$. Thus $\mathcal{I}_{M_{p}}^{-1}(A) \in L_{\left(\omega_{1}, \omega_{2}\right)}^{\prime\left(M_{p}\right)}\left(\Gamma_{v_{1}}^{1} \times \Gamma_{v_{2}}^{2}\right)$.

Next, repeating the proof of Theorem 1 in [6] we obtain that the operator $\mathcal{I}_{M_{p}}^{-1}$ is continuous and satisfies

$$
\mathcal{I}_{M_{p}} \mathcal{I}_{M_{p}}^{-1}=\operatorname{Id}_{L_{\left(\omega_{1}\right)}^{\prime\left(M_{p}\right)}\left(\Gamma_{v_{1}}^{1}, L_{\left(\omega_{2}\right)}^{\prime\left(M_{p}\right)}\left(\Gamma_{v_{2}}^{2}\right)\right)} \quad \text { and } \quad \mathcal{I}_{M_{p}}^{-1} \mathcal{I}_{M_{p}}=\operatorname{Id}_{L_{\left(\omega_{1}, \omega_{2}\right)}^{\prime\left(M_{p}\right)}\left(\Gamma_{v_{1}}^{1} \times \Gamma_{v_{2}}^{2}\right)}
$$

and the proof is complete.

\section{References}

[1] A. Beurling, Quasi-analyticity and general distributions, Lectures 4 and 5, AMS Summer Institute, Stanford, 1961.

[2] U. Franken, Continuous linear extension of ultradifferentiable functions of Beurling type, Math. Nachr. 164 (1993), 119-139.

[3] H. Komatsu, Ultradistributions, I. Structure theorems and a characterization, J. Fac. Sci. Univ. Tokyo 20 (1973), 25-105. 
[4] H. Komatsu, Ultradistributions, II. The kernel theorem and ultradistributions with support in a submanifold, J. Fac. Sci. Univ. Tokyo 24 (1977), 607-628.

[5] G. Eysik, Laplace ultradistributions on a half line and a strong quasi-analyticity principle, Ann. Polon. Math. 63 (1996), 13-33.

[6] S. Michalik, The kernel theorem for Laplace ultradistributions, Ann. Polon. Math. 77 (2001), 209-217.

[7] C. Roumieu, Sur quelques extensions de la notion de distribution, Ann. Sci. École Norm. Sup. 77 (1960), 47-121.

[8] Z. Szmydt and B. Ziemian, The Mellin Transformation and Fuchsian Type Partial Differential Equations, Kluwer, Dordrecht, 1992.

[9] V. S. Vladimirov, Generalized Functions in Mathematical Physics, Mir Publishers, Moscow, 1979 . 
